\title{
Cross-Validated Wavelet Block Thresholding for Non-Gaussian Errors
}

\author{
K.McGinnity ${ }^{\mathrm{a}}$, R.Varbanov ${ }^{\mathrm{b}, *}$, E.Chicken ${ }^{\mathrm{b}}$ \\ ${ }^{a}$ Institute for Defense Analyses, 4850 Mark Center Drive, Alexandria, Virginia 22311, USA \\ ${ }^{b}$ Department of Statistics, Florida State University, 117 N. Woodward Ave., Tallahassee, FL 32306, USA
}

\begin{abstract}
Wavelet thresholding generally assumes independent, identically distributed normal errors when estimating functions in a nonparametric regression setting. VisuShrink and SureShrink are just two of the many common thresholding methods based on this assumption. When the errors are not normally distributed, however, few methods have been proposed. A distribution-free method for thresholding wavelet coefficients in nonparametric regression is described, which unlike some other non-normal error thresholding methods, does not assume the form of the nonnormal distribution is known. Improvements are made to an existing even-odd cross-validation method by employing block thresholding and level dependence. The efficiency of the proposed method on a variety of non-normal errors, including comparisons to existing wavelet threshold estimators, is shown on simulated data.
\end{abstract}

Keywords: wavelets, thresholding, nonparametric function estimation

\section{Introduction}

Wavelet thresholding has been a staple of statistical functional estimation for years. Donoho and Johnstone (1994, 1995, 1998) introduced methods for thresholding the wavelet coefficients derived from the wavelet transformation of the observed data in nonparametric regression:

$$
y_{i}=f\left(x_{i}\right)+\varepsilon_{i}, \quad i=1,2, \ldots, n,
$$

where the $\varepsilon_{i}$ are independent and identically distributed (iid) Gaussian errors with mean zero and constant variance $\sigma^{2}$ and the sample points $x_{i}=i / n$ are equally spaced over an interval. By thresholding observed wavelet coefficients representing noise, a smooth estimate of the underlying can be obtained. The assumptions on the errors have been loosened in only a handful of papers on wavelet thresholding.

Neumann and von Sachs (1995) discuss wavelet thresholding methods in non-Gaussian and non-iid situations. The main idea of their paper is that, in many situations, asymptotic normality can be proven and traditional thresholding methods can be used. Given independent observations, they demonstrate a way to show equivalence to the Gaussian case via strong approximations. They also derive asymptotic normality in the case of weak dependence.

Antoniadis and Fryzlewicz (2006) propose a scale-dependent wavelet thresholding procedure for Gaussian noise, and then extend it to include non-Gaussian noise. However, the paper assumes not only that the non-normal errors are iid with mean zero, but also that they follow a known specified distribution. They determine a suitable threshold for each resolution level by mimicking the arguments of Donoho and Johnstone in the Gaussian case.

${ }^{*}$ Corresponding author

Email address: r.varbanov@stat.fsu.edu (R.Varbanov) 
Pensky and Sapatinas (2007) investigate the performance of Bayes factor estimators in wavelet regression models with iid non-Gaussian errors. They choose a general distribution $\eta_{j}$ for the errors and assume they possess symmetric PDFs on the reals that are unimodal, positive, and finite at zero. One advantage of their method is that knowledge of the true distribution of the errors is not needed in order to obtain an optimal estimator of $f$. However, their estimators are only preferable for irregular functions with high peaks, and produce sub-optimal results when compared with other methods under certain prior distributions.

Nason (1996) introduces an even-odd cross-validation method for choosing the threshold parameter in wavelet shrinkage. His statistic compares an interpolated wavelet estimator from the even reconstructed data to the odd noisy data and vice versa over various threshold values, then applies a sample size correction.

In this paper, we propose a completely nonparametric method to threshold wavelet coefficients that enhances Nason's cross-validation method by incorporating level-dependent block thresholding. Block thresholding thresholds wavelet coefficients in groups, rather than individually, with the goal of increasing precision by utilizing information about neighboring coefficients (Cai, 1999). Nason's method uses term-by-term thresholding, so it is reasonable to ask if incorporating blocking will have an analogous effect here.

Nason also makes use of a global threshold, the same threshold value for all considered coefficients. This is similar to VisuShrink of Donoho and Johnstone (1994). However, level-dependent thresholding has also been shown to have advantages over universal thresholds. For example, SureShrink (Donoho and Johnstone, 1995), a level-dependent thresholding method, has been shown to have lower mean squared error (MSE) than VisuShrink. Each of these modifications, blocking and level dependence, improves performance with distribution-based thresholds and are thus natural considerations for attempting to improve cross-validation thresholding.

Our method does not put any assumptions on the errors except that they are iid and centered at zero. Unlike Neumann and von Sachs (1995), we do not discuss asymptotic normality, but instead develop a method specifically meant to handle non-Gaussian errors. Nor do we require that the distribution of the errors be known, as do Antoniadis and Fryzlewicz (2006). Unlike that of Pensky and Sapatinas (2007), no proper choice of prior is required for our method.

This paper is divided as follows. Section 2 provides a brief background on wavelets, wavelet notation, and wavelet thresholding methods before the details of the proposed estimator are described in Section 3. Section 4 contains a simulation comparison of the proposed estimator to the Nason estimator, VisuShrink, and other current methods which may assume normal errors. A discussion of the results and methods is given in the final section.

\section{Background}

\subsection{Wavelets}

Wavelets are an orthogonal series representation of functions in the space of square-integrable functions $L_{2}(\mathbb{R})$. Ogden (1997) and Vidakovic (1999) offer good introductions to wavelet methods and their properties. Let $\phi$ and $\psi$ represent the father and mother wavelet functions, respectively. There are many choices for these two functions, see Daubechies (1992). Here, $\phi$ and $\psi$ are chosen to be compactly supported and to generate an orthonormal basis. Let

$$
\phi_{j k}(x)=2^{j / 2} \phi\left(2^{j} x-k\right)
$$

and

$$
\psi_{j k}(x)=2^{j / 2} \psi\left(2^{j} x-k\right)
$$

be the translations and dilations of $\phi$ and $\psi$, respectively. For any fixed integer $j_{0}$,

$$
\left\{\phi_{j_{0} k}, \psi_{j k} \mid j \geq j_{0}, k \text { an integer }\right\}
$$


is an orthonormal basis for $L_{2}(\mathbb{R})$. Let

$$
\xi_{j k}=\left\langle f, \phi_{j k}\right\rangle
$$

and

$$
\theta_{j k}=\left\langle f, \psi_{j k}\right\rangle
$$

be the usual inner product of a function $f \in L_{2}(\mathbb{R})$ with the wavelet basis functions. Then $f$ can be expressed as an infinite series:

$$
f(x)=\sum_{k} \xi_{j_{0} k} \phi_{j_{0} k}(x)+\sum_{j=j_{0}}^{\infty} \sum_{k} \theta_{j k} \psi_{j k}(x) .
$$

The function $f$ is not known and must be estimated. This is done using the discrete wavelet transform (DWT) of Mallat (1999). If $f$ is sampled as a vector of dyadic length $n=2^{J}$ for some positive integer $J$, then the DWT will provide a total of $n$ estimated coefficients $\xi_{j_{0} k}$ and $\theta_{j k}$ over the indices $j=j_{0}, j_{0}+1, \ldots, J-1$ and for all appropriate $k$. The lowest level possible for $j_{0}$ is 0 , the highest is $J-1$.

The wavelet basis functions are easily periodized to a specified interval. In this paper, we use wavelets that have been periodized to the interval $[0,1]$. In this case, the index $k$ for resolution level $j$ runs from 1 to $2^{j}$ in $(2)$.

Wavelets have the useful property that they can simultaneously analyze a function in both time and frequency. This is done by projecting the function to be analyzed into several subspaces or resolution levels. Each resolution level represents a different degree of smoothness of the function. The lowest resolution level, associated with the index $j=j_{0}$, represents the smoothest or coarsest part of the function. Increasing the index $j$ corresponds to decreasing smoothness. The highest resolution levels $j$ therefore represent the behavior of the function at the highest frequencies or scales. Since the wavelet series (2) forms an orthogonal representation, the sum of the projections in these resolution levels is the original function $f$. The construction of wavelet functions $\phi$ and $\psi$ provide the ability to localize the analysis within each subspace. The higher the resolution, the greater the degree of localization.

By varying the resolution level $j$, wavelets have the ability to zoom in or out onto the smooth or detailed structure of $f$. This is referred to as the multiresolution property of wavelets. Changing the index $k$ allows wavelets to localize the analysis. These properties enable wavelets to model functions of very irregular types, as well as smooth functions.

We use $W$ to denote the $n \times n$ DWT transformation matrix. Applying the DWT to the observed values $y=\left(y_{1}, y_{2}, \ldots, y_{n}\right)^{\prime}$ in (1) gives the estimated wavelet coefficients

$$
\tilde{\theta}=\left(\tilde{\xi}_{j_{0} 1}, \tilde{\xi}_{j_{0} 2}, \ldots, \tilde{\xi}_{j_{0} 2^{j_{0}}}, \tilde{\theta}_{j_{0} 1}, \tilde{\theta}_{j_{0} 2}, \ldots, \tilde{\theta}_{J-1,2^{J-1}}\right)^{\prime}=W y
$$

Applying the inverse DWT $W^{-1}=W^{\prime}$ to these coefficients returns the original data, $y=W^{\prime} W y$.

\subsection{Thresholding}

Most wavelet analysis uses some form of thresholding. These can be term-by-term methods, where each individual wavelet coefficient is modified individually, or block methods, where coefficients are modified in groups. There are two general types of term-by-term thresholding methods. Hard thresholding is a "keep-or-kill" operation that sets to zero any wavelet coefficient below a certain threshold value $\lambda$. In soft thresholding, coefficients smaller in magnitude than $\lambda$ are set to zero and coefficients larger than $\lambda$ are shrunk towards zero.

A well-known example of term-by-term thresholding is the VisuShrink method of Donoho and Johnstone (1994). For a single estimated coefficient $\tilde{\theta}_{j k}$,

$$
\hat{\theta}_{j k}=\eta\left(\tilde{\theta}_{j k}, \lambda\right)=\operatorname{sgn}\left(\tilde{\theta}_{j k}\right)\left(\left|\tilde{\theta}_{j k}\right|-\lambda\right)_{+}
$$


is the thresholded coefficient, where $\lambda$ is a threshold parameter. This is an example of soft thresholding. Hard thresholding via Visushrink can also be used. The value of $\lambda$ is chosen to give optimal results in terms of reconstruction; $\lambda=\sigma \sqrt{2 \log (n)}$ where $\sigma$ and $n$ are from (1). This is known as the universal threshold since the same threshold value is used across all resolution levels. A thresholded estimate is then formed as

$$
\hat{f}=W^{\prime} \eta(W y, \lambda)
$$

Note that only select resolution levels are subjected to thresholding. Typically these are the highest $m$ resolution levels, where $m$ is specified by the user.

Another popular thresholding method combines VisuShrink with a resolution-level-dependent method based on Stein's Unbiased Risk Estimator (SURE), see Stein (1981). This SURE-based method obtains optimal MSE but does not work well if the detail coefficients at a given resolution level are too sparse. Thus, this hybrid method will implement the universal threshold if a level is too sparse, and will use the SURE-based method otherwise. Level $j$ is too sparse if

$$
2^{-j} \sum_{k=1}^{2^{j}}\left(d_{j, k}^{2} / \sigma_{j, k}^{2}-1\right) \leq 2^{-j / 2}\left(\log _{2} 2^{j}\right)^{3 / 2} .
$$

One may choose to ignore the sparsity condition. We will refer to this as SureShrink in our paper, see Donoho and Johnstone (1995). Both the hybrid method (HybridShrink) and SureShrink are only designed to be used with soft thresholding, and are more computationally expensive than VisuShrink. Johnstone and Silverman (1997) further utilize the benefits of leveldependent thresholding in the handling of correlated noise as changes in the variance of observed wavelet coefficients as a result of this dependence will only occur between rather within levels.

Block thresholding methods typically employ variants of the block projection estimator of Cai (1999). If $B_{i}, i=1, \ldots, N$ are blocks of equal size $L(\sim \log n)$ that evenly divide the observed wavelet coefficients $\tilde{\theta}, \mathscr{H}$ is a subset of the block indices $\{1, \ldots, N\}$, and $\tilde{\theta}_{B_{i}}$ are the $L$ coefficients in block $B_{i}$, the goal is to have $\mathscr{H}$ consist only of those blocks where the signal is greater than the noise:

$$
\mathscr{H}=\mathscr{H}(\tilde{\theta})=\left\{i:\left\|\tilde{\theta}_{B_{i}}\right\|_{2}^{2}>L \sigma^{2}\right\},
$$

where $\left\|\tilde{\theta}_{B_{i}}\right\|_{2}^{2}=\sum_{k \in B_{i}} \tilde{\theta}_{k}^{2}$.

Anything less than the threshold is set to zero, giving the final block projection estimator to be

$$
\hat{\theta}_{B_{i}}=\left\{\begin{array}{cc}
\tilde{\theta}_{B_{i}} & \text { if } i \in \mathscr{H} \\
0 & \text { if } i \notin \mathscr{H}
\end{array}\right.
$$

So, in any particular block, either the original coefficients are kept, or every coefficient in the block is set to zero. Though blocking can also be implemented with soft thresholding as done by Wang and Wood (2006), who propose an Empirical Bayes block-dependent threshold in the presence of normal noise.

In more recent advances, Barber and Nason (2004) build on the multiwavelet shrinkage method introduced by Downie and Silverman (1996) by implementing the use of a complex wavelet transform to estimate real signals. The Complex Multiwavelet Style (CMWS) shrinkage method uses the complex-valued Daubechies wavelets used by Lawton (1993) and Lina and Mayrand (1995) and discards the imaginary component of the reconstructed signal. For complexvalued empirical wavelet coefficient $d_{j, k}^{*}$, where $d_{j, k}^{*} \sim N_{2}\left(d_{j, k}, \Sigma_{j}\right)$, a "thresholding statistic"

$$
\theta_{j, k}=d_{j, k}^{* T} \Sigma_{j}^{-1} d_{j, k}^{*}
$$


is computed. Hard thresholding is then carried out according to the rule

$$
\widehat{d}_{j, k}^{M H}=d_{j, k}^{*} I\left(\theta_{j, k}>\lambda\right)
$$

where $I$ is the indicator function and $\lambda=2 \log n$, a theshold derived by Downie and Silverman (1996) for use in the multiwavelet case.

Fryzlewicz (2007) combines thresholding and the unbalanced Haar (UH) basis introduced by Girardi and Sweldens (1997), for which jumps in the basis functions do not necessarily occur in the middle of their support. Each basis vector for the discrete UH transform is selected to best match the data at a specific scale and location by applying the idea of a matching pursuit algorithm originally introduced by Mallat and Zhang (1993). The author focuses on a top-down version of algorithm where the greatest concentration of the power of signal is placed on the coarse scales before proceeding to finer scales. After transformation, hard thresholding can be carried out by comparing the absolute value of empirical wavelet coefficient $Y_{j, k}$ to the universal threshold used in Visushrink, $\lambda=\sigma \sqrt{2 \log n}$.

Johnstone and Silverman (2005) propose the selection of a level-dependent threshold utilizing Empirical Bayes methods. Acknowledging the need for a threshold to adapt to the sparsity of a signal and consequently the resolution level, this Empirical Bayes method models this sparsity by selecting a suitable prior distribution for the wavelet coefficients of the signal. At each resolution level, the weights of the distributions that comprise the mixture prior are estimated using marginal-maximum likelihood. A threshold is calculated such that the posterior median of the distribution of the coefficients is zero if and only if the magnitude of the coefficients is less than said threshold. Hard thresholding can then be applied using the selected threshold level.

It is important to note that all the methods mentioned above put strong assumptions on the errors, particularly normality. Oh et al. (2007) propose an iterative ES-algorithm that can be combined with a thresholding selection method to create a robust wavelet regression procedure; they specifically advocate for the use of the aforementioned Empirical Bayes threshold selection of Johnstone and Silverman (2005). Their procedure relies on the transformation of observed data into pseudo data that define an additive model with a light-tailed random component suitable for traditional wavelet thresholding.

The properties and advantages of differing types of thresholding methods are well documented in the literature. See Donoho and Johnstone (1994, 1998); Cai (1999); Pensky (1999); Cai and Silverman (2001); Chicken (2003, 2005).

\section{The Estimator}

We observe a vector of data, $y_{i}=f\left(x_{i}\right)+\epsilon_{i}, i=1, \ldots, n$ for which the noise $\epsilon_{i}$ is independent and identically distributed. If the noise is not centered at zero, we subtract the mean of the noise from each $\epsilon_{i}$ to eliminate any potential bias. Specific choices for the noise will be discussed in more detail later. When the discrete wavelet transform (DWT) is applied to the data, we obtain $W y=W f(x)+W \epsilon$ where $W$ represents the wavelet transformation. If the original errors $\epsilon$ have mean zero and covariance $\Sigma=\sigma^{2} I_{n}$ then, since $W$ is an orthogonal transformation, the new errors $W \epsilon=\epsilon^{\prime}$ are uncorrelated. They are not necessarily independent unless the $\epsilon_{i}$ have a Gaussian distribution. Opsomer et al. (2001) show that issues in nonparametric regression only arise with correlated errors. The errors are also identically distributed within each resolution level, see McGinnity and Chicken (2012).

Our approach is motivated by Nason's even-odd cross-validation procedure. We propose three major modifications. First, we make the threshold level-dependent, meaning we allow a different threshold value to be chosen for each resolution level, rather than choosing a global threshold for all resolution levels. This provides increased estimability and accuracy. For example, SureShrink, which has a level-dependent threshold, has lower MSE than VisuShrink, which 
has a global threshold, but SureShrink often has noisier reconstructions. Second, we employ block thresholding. Block thresholding divides the data into blocks of neighboring coefficients, and will set to zero an entire block if the sum of the squared coefficients in that block is less than the threshold value. Block thresholding has been shown to have improved error and visual fit over traditional term-by-term thresholding (Cai, 1999), alleviating the above concern associated with balancing the smoothness and MSE of the reconstruction. Third, we add an additional term to the error statistic that gets minimized during cross-validation. This term compares two sampled reconstructions to one another.

To set up our algorithm, we first divide the noisy data of length $n$ into even and odd parts, $y^{e}$ and $y^{o}$ :

$$
y^{e}=\left(y_{2}, y_{4}, \ldots, y_{n}\right)^{\prime}
$$

and

$$
y^{o}=\left(y_{1}, y_{3}, \ldots, y_{n-1}\right)^{\prime} .
$$

We then apply the DWT to each part to obtain $\hat{f}^{e}$ and $\hat{f}^{o}$. We choose the block size to be the closest dyadic number to $\log (n)$ (shown to possess optimal properties in Cai (1999)). We define the threshold search range to be zero to the maximum sum of squared coefficients in any block. This is the most conservative search range we can choose since the minimum threshold will set all detail coefficients to be zero, giving the smoothest possible reconstruction, and the maximum threshold will set nothing to zero and return the original noisy data. It is possible to reduce the size of this search range through methods such as pilot density estimation on coefficients in the first resolution level, however, in doing so, one risks leaving out the optimal threshold value from the search.

In the first iteration, we start by finding the optimal threshold value $\lambda$ for all detail resolution levels simultaneously by minimizing a combination of two errors. The first error is the sum of the squared difference between the odd reconstruction and the even data, and the squared difference between the even reconstruction and the odd data. This mimics the method of Nason (1998), except we apply the threshold rule to all coefficients in a block rather than individual coefficients. The second error is the sum of the squared difference between the odd reconstruction and the even reconstruction. This error is not considered by Nason. We choose to use this combination of errors in order to obtain the optimal smoothness. Intuitively, we expect a comparison between the noisy data and the smooth reconstructions to under-smooth our estimate as it equally balances an already reasonable estimate of function and the data itself which we know to be contaminated with noise; similarly, we expect a comparison of the the even and odd reconstructions to over-smooth our estimate as it does not directly incorporate the original observations themselves. Thus, the initial global threshold estimate is

$$
\hat{\lambda}=\underset{\lambda}{\operatorname{argmin}}\left\{\frac{1}{2}\left\{\left\|\hat{f}_{\lambda}^{o}-y^{e}\right\|_{2}^{2}+\left\|\hat{f}_{\lambda}^{e}-y^{o}\right\|_{2}^{2}\right\}+\frac{1}{2}\left\{\left\|\hat{f}_{\lambda}^{o}-\hat{f}_{\lambda}^{e}\right\|_{2}^{2}\right\}\right\} .
$$

We then proceed to make our estimator level-dependent. Letting $\lambda_{j}$ be the threshold value for resolution level $j\left(j=j_{0}, \ldots, J-1\right)$, we fix $\lambda_{J-1}$ at the value found in (7) and repeat the search described above to find an optimal threshold for resolution levels $J-2, \ldots, j_{0}$. This value becomes our $\lambda_{J-2}$ and we next search for the optimal threshold for resolutions levels $J-3, \ldots, j_{0}$. This value becomes our $\lambda_{J-3}$ and we then find the optimal threshold for resolution level $J-4, \ldots, j_{0}$. Repeat this process until the optimal $\lambda_{j_{0}}$ for $j_{0}$ alone is found. These serve as our initial level-dependent threshold estimates.

In the second and all subsequent iterations, we modify each resolution level threshold value one at a time until convergence is achieved. First, fix $\lambda_{J-2}, \lambda_{J-3}, \ldots, \lambda_{j_{0}}$ at their previous iteration values and search for the optimal $\lambda_{J-1}$. Next, fix $\lambda_{J-1}$ at this new value and $\lambda_{J-3}, \ldots, \lambda_{j_{0}}$ at their previous iteration values and search for the optimal $\lambda_{J-2}$. Then, fix $\lambda_{J-1}$ and $\lambda_{J-2}$ at their 
new values and $\lambda_{J-4}, \ldots, \lambda_{j_{0}}$ at their previous iteration values and search for the optimal $\lambda_{J-3}$. Continue until all $\lambda_{j}$ have been modified individually. We found that this process generally only had to be performed once before all threshold values converged.

The last step in our procedure is also a modification of Nason's final step. Since all estimates in his paper were based on $n / 2$ data points, a heuristic method based on the universal threshold was used to obtain a threshold for $n$ data points. Once his error statistic had been minimized to obtain a threshold value $\lambda^{n / 2}$, the following correction was applied to obtain the final threshold:

$$
\lambda^{n} \approx\left(1-\frac{\log 2}{\log n}\right)^{-1 / 2} \cdot \lambda^{n / 2} .
$$

where the superscript refers to the number of points used to obtain the threshold value.

In our case, the sample size used to calculate the statistic changes depending on which resolution level $j$ we are working with ( $j=1$ representing the highest resolution level). Thus we apply the following level-dependent correction:

$$
\lambda_{j}^{n / 2^{j}} \approx\left(1-\frac{\log 2}{\log \left(\frac{n}{2^{j}}\right)}\right)^{-1} \cdot \lambda_{j}^{n / 2^{j+1}}, j=1,2,3,4, \ldots, J-j_{0}
$$

Notice that we no longer need to take the square root in our correction since our threshold values are based on the sum of squared coefficients within a block. Again, the superscript of $\lambda$ refers to the number points used to obtain the threshold value and does not indicate an exponent.

\section{Simulation}

Our estimator is compared to the traditional thresholding method of VisuShrink, Nason's method, the CMWS shrinkage of Barber and Nason (2004), the UH technique of Fryzlewicz (2007), the Empirical Bayes (EBayes) selection of thresholds of Johnstone and Silverman (2005), and the Robust Empirical Bayes thresholding (REBayes) of Oh et al. (2007) via MSE. (See McGinnity and Chicken (2012) for comparisons to Antoniadis' method.)

Using sample sizes ranging from $2^{9}$ to $2^{11}$ and signal-to-noise ratios (SNRs) of 3 and 5 , these methods were tested on the eight standard test functions (Blip, Blocks, Bumps, Corner, Doppler, Heavisine, Spikes, and Wave), see Donoho and Johnstone (1994) and Marron et al. (1998). Figure 1 shows a plot of these functions.

Various types of noise were added on top of these test functions to create our data. The only assumptions imposed on the errors were that they were independent and identically distributed. In this paper we show results for $T_{3}$ and lognormal errors. Other types of noise were considered, but these two were chosen to exemplify a heavy-tailed distribution and a skewed distribution. The noise was scaled appropriately to obtain the desired SNR, thus the true distribution of the noise was not necessarily known. For example, a scaled $t$-distribution is no longer a $t$. In the case of skewed distributions such as lognormal, we first centered the noise at the mean before applying it to the test function. Figure 2 shows a plot of $T_{3}$ noise added to all the test functions. 

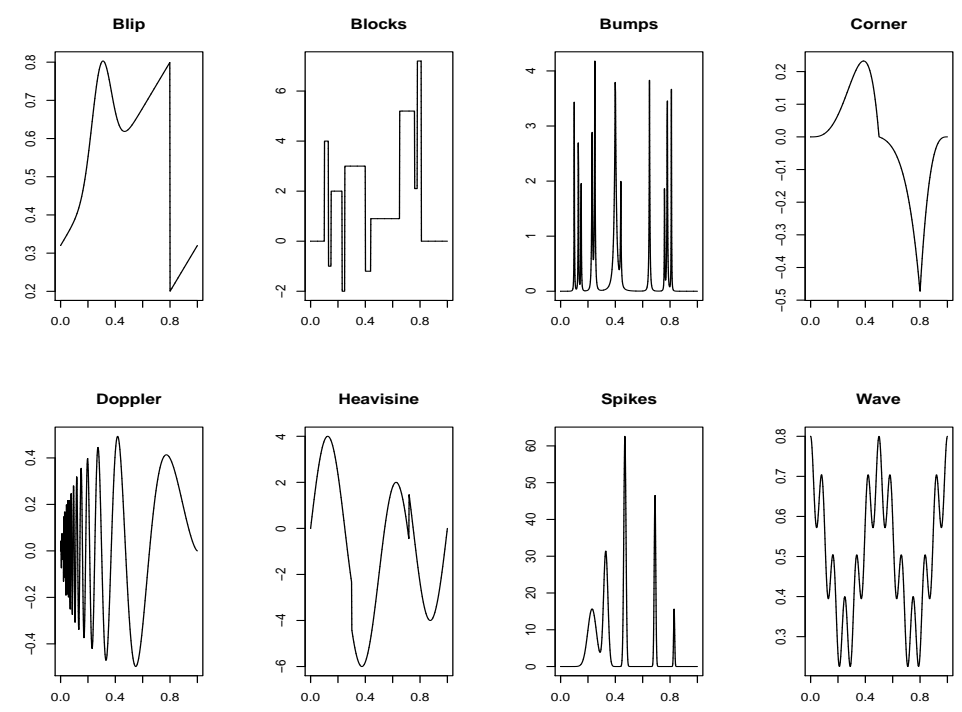

Figure 1: The eight test functions used in this paper sampled at 512 points.
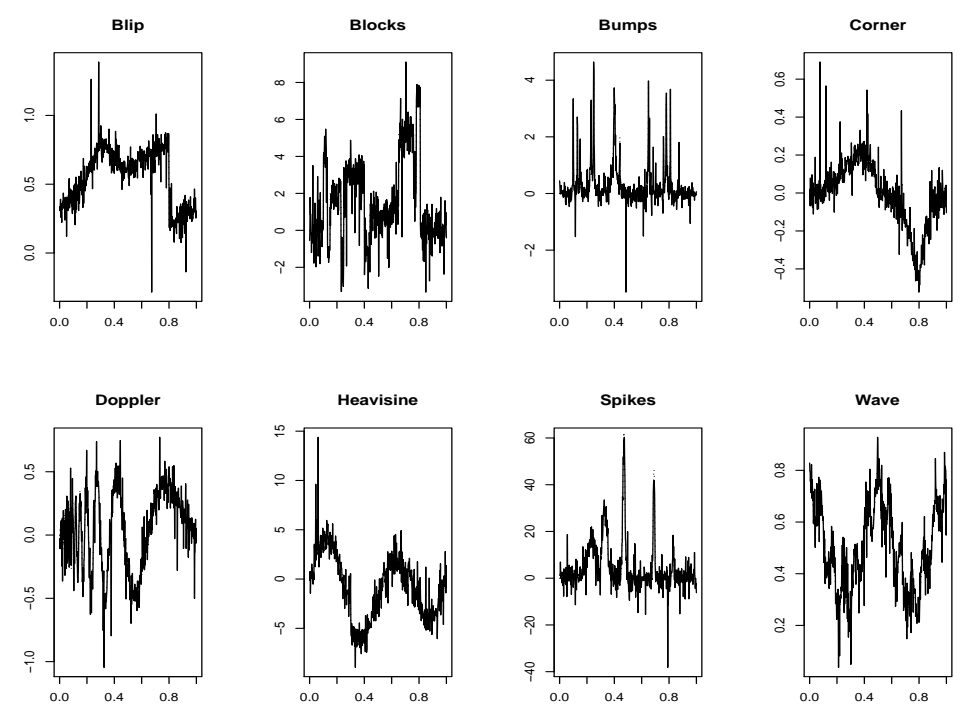

Figure 2: The test functions with scaled $T_{3}$ noise added to represent a SNR of 5 .

When possible, all functions were analyzed using the least asymmetric wavelet basis of length eight (LA-8) (Daubechies, 1992) with periodic boundary handling, and the lowest resolution level, $j_{0}$, set to $J-4$. For the CMWS method, estimation was done using the complex-valued Daubechies wavelet basis with four vanishing moments. For the UH method, the functions were analyzed with the best top-down UH decomposition. For the EBayes method, a Laplace prior was used for the wavelet coefficients at each level. For the REBayes method, the EBayes method as described above was implemented for threshold selection and $\log (\cosh )$ was used as $\rho$ in the formation of the pseudo data. All simulations were performed in R (R Development Core Team, 2009) using the waveslim (Whitcher, 2009), Wave Thresh (Nason, 2013), unbalhaar (Fryzlewicz, 2010), and EBayes Thresh (Silverman, 2010) packages.

Tables 1 and 2 show simulation results of 100 repetitions each for $T_{3}$ and lognormal noise with SNR=5 (see McGinnity et al. (2016) for full results). Hard thresholding and various combinations of $n$ and test function were used. The numbers in the table represent the ratio 
of the average MSE for the given method to the average MSE of VisuShrink. Thus a ratio less than one indicates that a particular method outperformed VisuShrink, while a ratio greater than 1 indicates that VisuShrink performed better. For each combination of conditions, we have identified the best performing method by highlighting the lowest average MSE. We also highlighted any other average MSE that was not significantly different than the lowest average MSE according to a paired $t$-test at the $\alpha=0.05$ significance level.

Table 1: $T_{3}$ noise with $\mathrm{SNR}=5$ : Ratio of average MSE of listed method to average MSE of VisuShrink

\begin{tabular}{cccccccc}
\hline Function & $\mathrm{n}$ & LD Block & Nason & CMWS & UH & EBayes & REBayes \\
\hline \multirow{3}{*}{ Blip } & 512 & 0.60 & 0.47 & 1.06 & 1.22 & 1.35 & 2.01 \\
& 1024 & 0.43 & 0.49 & 1.02 & 1.14 & 1.40 & 2.10 \\
& 2048 & 0.29 & 0.36 & 1.02 & 1.10 & 1.44 & 2.15 \\
\hline \multirow{3}{*}{ Blocks } & 512 & 1.45 & 1.10 & 0.93 & 0.61 & 1.08 & 0.83 \\
& 1024 & 0.88 & 0.85 & 0.95 & 0.71 & 1.21 & 1.01 \\
& 2048 & 0.57 & 0.64 & 0.93 & 0.78 & 1.28 & 1.05 \\
\hline \multirow{3}{*}{ Bumps } & 512 & 1.68 & 2.09 & 0.76 & 0.92 & 0.99 & 1.10 \\
& 1024 & 1.33 & 2.12 & 0.88 & 1.18 & 1.23 & 1.47 \\
& 2048 & 0.84 & 1.18 & 0.93 & 1.27 & 1.33 & 1.72 \\
\hline \multirow{3}{*}{ Corner } & 512 & 0.20 & 0.25 & 1.04 & 1.35 & 1.34 & 2.05 \\
& 1024 & 0.19 & 0.24 & 1.04 & 1.29 & 1.41 & 2.22 \\
& 2048 & 0.19 & 0.25 & 1.02 & 1.18 & 1.44 & 2.21 \\
\hline \multirow{3}{*}{ Heavisine } & 512 & 0.91 & 0.83 & 0.85 & 1.50 & 1.19 & 1.49 \\
& 1024 & 0.42 & 0.73 & 0.95 & 1.53 & 1.34 & 1.83 \\
& 2048 & 0.43 & 0.47 & 0.98 & 1.41 & 1.41 & 1.99 \\
\hline \multirow{3}{*}{ Spikes } & 512 & 0.21 & 0.26 & 1.06 & 1.44 & 1.35 & 1.18 \\
& 2048 & 0.22 & 0.26 & 1.03 & 1.33 & 1.40 & 1.27 \\
& 512 & 0.20 & 0.26 & 1.02 & 1.21 & 1.44 & 1.27 \\
\hline \multirow{3}{*}{ Wave } & 1024 & 0.46 & 0.56 & 0.99 & 1.31 & 1.39 & 1.34 \\
& 2048 & 0.22 & 0.27 & 1.01 & 1.25 & 1.43 & 1.36 \\
\hline & 512 & 0.46 & 0.47 & 0.86 & 1.39 & 1.07 & 1.51 \\
& 2048 & 0.21 & 0.25 & 1.04 & 1.63 & 1.41 & 2.20 \\
& 0.19 & 0.25 & 1.02 & 1.41 & 1.44 & 2.20 \\
\hline \multirow{3}{*}{ Dopler } & & & & & &
\end{tabular}


Table 2: Lognormal noise with $\mathrm{SNR}=5$ : Ratio of average MSE of listed method to average MSE of VisuShrink

\begin{tabular}{cccccccc}
\hline Function & $\mathrm{n}$ & LD Block & Nason & CMWS & UH & EBayes & REBayes \\
\hline \multirow{3}{*}{ Blip } & 512 & 0.37 & 0.31 & 1.06 & 1.24 & 1.34 & 1.51 \\
& 1024 & 0.23 & 0.31 & 1.04 & 1.21 & 1.35 & 1.51 \\
& 2048 & 0.17 & 0.21 & 1.04 & 1.20 & 1.39 & 1.56 \\
\hline \multirow{3}{*}{ Blocks } & 512 & 1.23 & 0.99 & 1.00 & 0.97 & 1.18 & 1.14 \\
& 1024 & 0.58 & 0.62 & 1.00 & 1.03 & 1.25 & 1.26 \\
& 2048 & 0.39 & 0.44 & 1.01 & 1.09 & 1.33 & 1.39 \\
\hline \multirow{3}{*}{ Bumps } & 512 & 1.56 & 1.97 & 0.91 & 1.04 & 1.13 & 1.19 \\
& 1024 & 0.93 & 1.55 & 0.98 & 1.15 & 1.25 & 1.33 \\
& 2048 & 0.58 & 0.85 & 1.01 & 1.21 & 1.34 & 1.46 \\
\hline \multirow{3}{*}{ Corner } & 512 & 0.12 & 0.17 & 1.05 & 1.27 & 1.33 & 1.51 \\
& 1024 & 0.11 & 0.15 & 1.05 & 1.24 & 1.35 & 1.53 \\
& 2048 & 0.11 & 0.14 & 1.04 & 1.22 & 1.39 & 1.57 \\
\hline \multirow{3}{*}{ Heavisine } & 512 & 0.71 & 0.67 & 0.99 & 1.32 & 1.26 & 1.37 \\
& 1024 & 0.25 & 0.49 & 1.02 & 1.28 & 1.31 & 1.44 \\
& 2048 & 0.26 & 0.29 & 1.02 & 1.27 & 1.37 & 1.51 \\
\hline \multirow{3}{*}{ Spikes } & 512 & 0.13 & 0.18 & 1.06 & 1.28 & 1.32 & 0.86 \\
& 2048 & 0.12 & 0.17 & 1.04 & 1.24 & 1.34 & 0.83 \\
& 512 & 0.11 & 0.15 & 1.04 & 1.23 & 1.39 & 0.83 \\
\hline \multirow{3}{*}{ Wave } & 024 & 0.25 & 0.36 & 1.03 & 1.21 & 1.32 & 0.71 \\
& 512 & 0.13 & 0.16 & 1.03 & 1.23 & 1.38 & 0.74 \\
\hline & 2048 & 0.11 & 0.35 & 0.96 & 1.24 & 1.19 & 1.33 \\
& & 0.11 & 0.16 & 1.04 & 1.33 & 1.35 & 1.52 \\
& & 0.14 & 1.04 & 1.29 & 1.39 & 1.57 \\
\hline \multirow{6}{*}{ Dopler } & & & & & &
\end{tabular}

We considered three different classifications for the results of the proposed method (LD Block). Wins are cases where LD Block had the lowest average MSE and this average MSE is significantly different than all other competitors in pairwise comparisons. Losses are cases where LD Block has a significantly different average MSE than the leader. Ties are cases where LD Block is not significantly different than the leader or LD Block is the leader but is not significantly different than all other methods.

For $T_{3}$ noise and $\mathrm{SNR}=5$, LD Block has 14 wins, 7 losses, and 3 ties. When $\mathrm{SNR}=3$, LD Block has 16 wins, 4 losses, and 4 ties. Regardless of SNR, the proposed method loses only three of all the comparisons at the highest two sample sizes $\left(n=2^{10}\right.$ and $\left.2^{11}\right)$. All cases in which the proposed method is significantly worse than the leading method involve either the smallest sample size or test functions Blocks and Bumps.

For lognormal noise and SNR=5, LD Block has 18 wins, 3 losses, and 3 ties. The only time a competitor beats the proposed method in terms of MSE is at the smallest sample size and test functions Blips, Blocks, and Bumps. When SNR=3, LD Block has 20 wins, 1 loss, and 3 ties.

LD Block, Nason, and CMWS show substantial improvement over VisuShrink, UH, EBayes and REBayes for both $T_{3}$ and lognormal noise, particularly for smoother functions. This improvement increases as SNR decreases. Clearly, outside of CMWS, traditional methods that assume normality fail drastically when given heavy-tailed or skewed errors.

The proposed method also provides very visually appealing reconstructions for both smooth and spiky functions. Figure 3 shows the average reconstruction over 10 repetitions for the proposed method and Nason's method using the Blip test function, $T_{3}$ noise, $\mathrm{SNR}=3$, and $n=512$. 
Both Nason's and the proposed method provide much smoother average reconstructions than does VisuShrink (see McGinnity and Chicken (2012) for VisuShrink reconstruction), however, it is clear that, in four or five places, Nason's method does not threshold coefficient(s) that it should have. This does not appear to happen at all for our level-dependent block method.
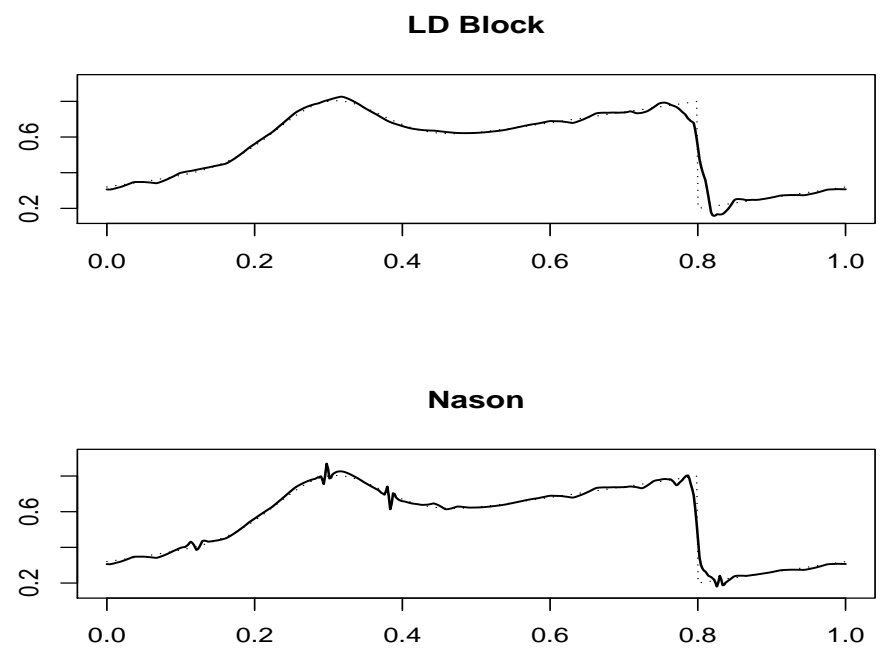

Figure 3: Average reconstructions for our method and Nason's method using the Blip test function with $T_{3}$ noise, $\mathrm{SNR}=3$, and $\mathrm{n}=512$

We tested the robustness of our method by comparing the performance of our estimator to current estimators with Gaussian errors. Table 3 shows simulation results of 100 repetitions for normal noise with $\mathrm{SNR}=5$ (see McGinnity et al. (2016) for full results). Our method is intended for use in situations when errors are thought to be non-normal, therefore we do not necessarily expect our method to be an improvement. For normal noise and SNR=5, LD Block has 2 wins, 17 losses, and 5 ties. For normal noise and SNR=3, LD block has 2 wins, 16 losses, and 6 ties. With Gaussian errors, CMWS appears to be the optimal method as it is significantly different than the lowest MSE in only 13 of 48 cases. In a head-to-head comparison, CMWS outperforms LD Block in 35 of 48 cases with normal noise and has an average MSE that is on average 0.58 times that of LD Block. However, in the 96 cases with non-normal errors, LD Block outperforms CMWS 85 times and has an average MSE that is on average 0.22 that of CMWS. We see that we outperform CMWS when we are expected to and we do so by a much wider margin than when CMWS outperforms our method. 
Table 3: Normal noise with $\mathrm{SNR}=5$ : Ratio of MSE of listed method to MSE of VisuShrink

\begin{tabular}{|c|c|c|c|c|c|c|c|}
\hline Function & $\mathrm{n}$ & LD Block & Nason & CMWS & $\mathrm{UH}$ & EBayes & REBayes \\
\hline \multirow{3}{*}{ Blip } & 512 & 2.04 & 1.07 & 1.10 & 1.38 & 1.18 & 4.27 \\
\hline & 1024 & 1.20 & 1.05 & 0.79 & 0.90 & 1.00 & 3.94 \\
\hline & 2048 & 1.14 & 1.00 & 0.94 & 0.81 & 1.02 & 5.21 \\
\hline \multirow{3}{*}{ Blocks } & 512 & 1.81 & 1.05 & 0.82 & 0.13 & 0.84 & 1.23 \\
\hline & 1024 & 1.40 & 1.05 & 0.82 & 0.10 & 0.87 & 1.85 \\
\hline & 2048 & 1.11 & 1.04 & 0.75 & 0.08 & 0.88 & 2.43 \\
\hline \multirow{3}{*}{ Bumps } & 512 & 1.76 & 1.94 & 0.55 & 0.86 & 0.71 & 0.90 \\
\hline & 1024 & 1.72 & 2.14 & 0.68 & 1.22 & 0.84 & 1.43 \\
\hline & 2048 & 1.53 & 0.94 & 0.68 & 1.50 & 0.87 & 2.18 \\
\hline \multirow{3}{*}{ Corner } & 512 & 0.98 & 1.08 & 1.00 & 2.67 & 1.29 & 5.66 \\
\hline & 1024 & 0.96 & 1.07 & 1.02 & 2.03 & 1.13 & 6.34 \\
\hline & 2048 & 0.97 & 1.03 & 0.98 & 1.39 & 1.06 & 6.40 \\
\hline \multirow{3}{*}{ Doppler } & 512 & 1.54 & 0.94 & 0.61 & 2.01 & 0.95 & 1.66 \\
\hline & 1024 & 0.83 & 0.95 & 0.71 & 2.38 & 0.96 & 2.64 \\
\hline & 2048 & 1.39 & 0.99 & 0.79 & 2.42 & 0.98 & 3.84 \\
\hline \multirow{3}{*}{ Heavisine } & 512 & 0.94 & 1.06 & 1.05 & 2.89 & 1.25 & 6.35 \\
\hline & 1024 & 0.96 & 1.06 & 0.97 & 2.29 & 1.12 & 7.07 \\
\hline & 2048 & 0.97 & 1.03 & 0.97 & 1.61 & 1.06 & 7.60 \\
\hline \multirow{3}{*}{ Spikes } & 512 & 2.04 & 0.95 & 0.59 & 1.40 & 0.98 & 2.84 \\
\hline & 1024 & 1.41 & 1.01 & 0.78 & 1.94 & 1.04 & 5.86 \\
\hline & 2048 & 0.98 & 1.03 & 0.93 & 1.97 & 1.06 & 8.67 \\
\hline \multirow{3}{*}{ Wave } & 512 & 0.97 & 1.01 & 0.62 & 2.18 & 0.82 & 2.04 \\
\hline & 1024 & 1.11 & 1.06 & 0.99 & 4.56 & 1.12 & 6.19 \\
\hline & 2048 & 0.97 & 1.03 & 0.98 & 2.99 & 1.06 & 6.39 \\
\hline
\end{tabular}

\section{Discussion and Conclusion}

Our goal in developing the proposed method was to provide a completely non-parametric way to threshold wavelet coefficients when the distribution of the noise is unknown. Simulation results show that our level-dependent block thresholding method is an improvement over the existing cross-validated method as well as traditional methods for various types of non-Gaussian noise.

Other combinations of cross-validation and block thresholding methods were also considered. Generally speaking, global block thresholding (Block) was approximately equivalent to Nason's method in terms of MSE, while the term-by-term level-dependent version of Nason's method (LD) was an improvement over Nason's global method (Nason) but not as good as the proposed level-dependent block method (LD Block). A version of the proposed method that was block dependent as opposed to level dependent (BD Block) was considered as well, but it did not perform very well. Since this method allows for a different threshold value to be chosen for every individual block, it was a very local analysis and was likely trying to model too much of the noise. It was also very computationally expensive. (See McGinnity and Chicken $(2012)$ for full results). When implemented separately with our cross-validation metric, level dependence was a more important modification to current methods than blocking. Our modified crossvalidation metric, when combined with blocking, did not significantly outperform the current cross-validation method. The general MSE trend for non-normal noise was:

LD Block $<\mathrm{LD}<$ Nason $\approx$ Block $<$ BD Block $<$ VisuShrink. 
An alternate cross-validation method was considered, in which we randomly chose (without replacement) half of the noisy data on which to perform the DWT and thresholding, and then compared that reconstruction to the other half of the data. However this method did not perform quite as well, and was significantly slower than the even-odd method, since numerous repetitions of the random selection and cross-validation were needed to ensure valid reconstructions.

Our method and its competitors could also be implemented using a non-decimated, or translation invariant, wavelet transform before thresholding. In practice, this may be appropriate as we would expect it to improve the MSE of each method, including ours. However, given the high computational cost of implementing such a transform, it was not a practical choice for our comparison given the number of different conditions and repetitions.

When considering the type of noise to add to our test functions, we intentionally chose distributions with finite variance. This way the SNR is defined and can be used as a measure of the amount of noise in our data. Any distribution with infinite variance, such as Cauchy, cannot be properly scaled, and has arbitrarily large errors.

Our analysis of the proposed method is extended in the technical report McGinnity et al. (2016). We expand our performance comparison to include two relevant real data examples and evaluate the results through visual comparisons of the reconstructions and analysis of threshold values. We also provide code and documentation to implement our method and generate the test functions used.

\section{Acknowledgments}

This research was supported by the Office of the Secretary of Defense, Directorate of Operational Test and Evaluation and the Test Resource Management Center under the Science of Test research program. Special thanks to both Dr. Catherine Warner, OSD DOT\&E, and Mr. George Rumford, TRMC, for the sponsorship of the research program.

\section{Disclaimer}

The views expressed in this article are those of the author and do not reflect the official policy or position of the Department of Defense or the U.S. Government. 


\section{References}

Antoniadis, A., Fryzlewicz, P., 2006. Parametric modelling of thresholds across scales in wavelet regression. Biometrika 93, 465-471.

Barber, S., Nason, G.P., 2004. Real nonparametric regression using complex wavelets. Journal of the Royal Statistical Society: Series B (Statistical Methodology) 66, 927-939.

Cai, T., 1999. Adaptive wavelet estimation: A block thresholding and oracle inequality approach. Ann. Statist. 27, 898-924.

Cai, T., Silverman, B., 2001. Incorporating information on neighboring coefficients into wavelet estimation. Sankhya Ser. B 63, 127-148.

Chicken, E., 2003. Block thresholding and wavelet estimation for nonequispaced samples. J. Statist. Plann. Inference 116, 113-129.

Chicken, E., 2005. Block-dependent thresholding in wavelet regression. Journal of Nonparametric Statistics 17, 467-491.

Daubechies, I., 1992. Ten Lectures on Wavelets. SIAM, Philadelphia.

Donoho, D., Johnstone, I., 1994. Ideal spatial adaptation via wavelet shrinkage. Biometrika 81, 425-455.

Donoho, D., Johnstone, I., 1995. Adapting to unknown smoothness via wavelet shrinkage. J. Am. Stat. Assoc. 90, 1200-1224.

Donoho, D., Johnstone, I., 1998. Minimax estimation via wavelet shrinkage. Annals of Statistics 26, 879-921.

Downie, T.R., Silverman, B.W., 1996. The discrete multiple wavelet transform and thresholding methods. IEEE Transactions in Signal Processing 46, 2558-2561.

Fryzlewicz, P., 2007. Unbalanced haar technique for nonparametric function estimation. Journal of the American Statistical Association 102, 1318-1327.

Fryzlewicz, P., 2010. unbalhaar: Function estimation via Unbalanced Haar wavelets. URL: https://cran.r-project.org/web/packages/unbalhaar/unbalhaar.pdf. r package version 2.0 .

Girardi, M., Sweldens, W., 1997. A new class of unbalanced haar wavelets that form an unconditional basis for $L_{p}$ on general measure spaces. Journal of Fourier Analysis and Applications $3,457-474$.

Johnstone, I.M., Silverman, B.W., 1997. Wavelet threshold estimators for data with correlated noise. Journal of the Royal Statistical Society: Series B (Statistical Methodology) 59, 319-351. URL: http://dx.doi.org/10.1111/1467-9868.00071, doi:10.1111/1467-9868.00071.

Johnstone, I.M., Silverman, B.W., 2005. Empirical bayes selection of wavelet thresholds. Annals of Statistics , 1700-1752.

Lawton, W., 1993. Applications of complex valued wavelet transforms to subband decomposition. IEEE Transactions in Signal Processing 41, 3566-3568.

Lina, J.M., Mayrand, M., 1995. Complex daubechies wavelets. Applied and Computaional Harmonic Analysis 2, 219-229. 
Mallat, S., 1999. A Wavelet Tour of Signal Processing. 3 ed., Academic Press, San Diego.

Mallat, S., Zhang, Z., 1993. Matching pursuit with time-frequency dictionaries. IEEE Transactions in Signal Processing 41, 3397-3415.

Marron, S., Adak, S., Johnstone, I., Neuman, M., Patil, P., 1998. Exact risk analysis of wavelet regression. J. Comput. Graph. Statist. 7, 278-309.

McGinnity, K., Chicken, E., 2012. Wavelet Block Thresholding for Non-Gaussian Errors. Technical Report M1006. Florida State University Department of Statistics. URL: http: //stat.fsu.edu/techreports/M1006.pdf.

McGinnity, K., Varbanov, R., Chicken, E., 2016. Cross-validated wavelet block thresholding for non-gaussian errors arXiv:1409.1130v3

Nason, G., 1996. Wavelet shrinkage using cross-validation. Journal of the Royal Statistical Society 58, 463-479.

Nason, G., 1998. WaveThresh3 Software. Department of Mathematics, University of Bristol, Bristol, UK.

Nason, G., 2013. wavethresh: Wavelets statistics and transforms. URL: https://cran. r-project.org/web/packages/wavethresh/wavethresh.pdf. r package version 4.6.6.

Neumann, M.H., von Sachs, R., 1995. Wavelet thresholding: Beyond the Gaussian I.I.D. situation, in: Lect. Notes Statist, Springer-Verlag. pp. 301-329.

Ogden, R., 1997. Essential Wavelets for Statistical Applications and Data Analysis. Birkhauser, Boston.

Oh, H.S., Nychka, D.W., Lee, T.C.M., 2007. The role of pseudo data for robust smoothing with application to wavelet regression. Biometrika 94, 893-904. URL: http://www.jstor.org/ stable/20441424.

Opsomer, J., Wang, Y., Yang, Y., 2001. Nonparametric regression with correlated errors. Statistical Science 16, 134-153.

Pensky, M., 1999. Estimation of a smooth density function using meyer-type wavelets. Statistics and Decisions 17, 111-123.

Pensky, M., Sapatinas, T., 2007. Frequentist optimatlity of Bayes factor estimators in wavelet regression models. Statistica Sinica 17, 599-633.

R Development Core Team, 2009. R: A Language and Environment for Statistical Computing. R Foundation for Statistical Computing. Vienna, Austria. URL: http://www.R-project.org. ISBN 3-900051-07-0.

Silverman, B.W., 2010. EbayesThresh: Empirical Bayes Thresholding and Related Methods. URL: https://cran.r-project.org/web/packages/EbayesThresh/EbayesThresh.pdf. r package version 1.3.2.

Stein, C., 1981. Estimation of the mean of a multivariate normal distribution. The Annals of Statistics 9, 1135-1151.

Vidakovic, B., 1999. Statistical Modeling by Wavelets. Wiley, New York. 
Wang, X., Wood, A.T.A., 2006. Empirical bayes block shrinkage of wavelet coefficients via the noncentral 2 distribution. Biometrika 93, 705-722. URL: http://search.ebscohost.com/ login aspx?direct=true\&db=a9h\&AN=22770393\&site=ehost-live.

Whitcher, B., 2009. waveslim: Basic wavelet routines for one-, two- and three-dimensional signal processing. URL: http://CRAN.R-project.org/package=waveslim. r package version 1.6.3. 